\title{
Factores que controlan la variabilidad morfológica de Pediastrum tetras bajo diferentes condiciones experimentales
}

\author{
Matilde Segura *, Carmen Rojo y María A. Rodrigo \\ Grupo de Ecología Integrativa. Instituto Cavanilles de Biodiversidad y Biología Evolutiva. Universidad de \\ Valencia. Apdo. Oficial 2085. E-46071 Valencia, España. \\ *Corresponding author: M.Matilde.Segura@uv.es
}

\begin{abstract}
Factors that control the morphologic variability of Pediastrum tetras under different experimental conditions

Morphological variability and plasticity in phytoplankton populations are important for taxonomy, systematic, and evolutionary studies and are key characteristics for ecological plankton processes. The green algae Pediastrum tetras (Ehrenberg) Ralfs was studied to describe the variation in its population size structure and its response to changes in biotic (presence of Daphnia magna (Straus) and its info-chemicals) and abiotic factors (nutrient concentration). The results indicated that the nutrient supply could affect the coenobium population structure (diminishing the most common coenobium, the 8celled one). D. magna significantly reduced $P$. tetras population density, because depredation affected it by significantly decreasing the biggest coenobium's density. In addition, info-chemicals induced a lower increase of the biggest coenobium when compared to the controls (rate of change was $45 \%$ lower than in control cultures). Our study suggests that $P$. tetras coenobium size is subjected to a possible compromise (trade-off) in their natural environment: $P$. tetras population would tend to increase their size for a better use of nutrients and to decrease it when big herbivores are present.
\end{abstract}

Key words: Coenobium formation, Daphnia magna, info-chemicals, trade-off.

\section{RESUMEN}

Factores que controlan la variabilidad morfológica de Pediastrum tetras bajo diferentes condiciones experimentales

La plasticidad y variabilidad morfológica en poblaciones de fitoplancton son importantes para estudios de taxonomía, sistemática y evolución y son características clave para procesos ecológicos del plancton. Se ha estudiado el alga verde Pediastrum tetras (Ehrenberg) Ralfs para describir la variación en la estructura de tamaños de la población y su respuesta frente a factores bióticos (presencia de Daphnia magna (Straus) y sus info-químicos) y abióticos (concentración de nutrientes). Los resultados indicaron que el suministro de nutrientes podría afectar la estructura de los cenobios de la población (disminuyendo el cenobio más común, el de 8 células). D. magna redujo significativamente la densidad de la población de P. tetras ya que la depredación le afectaba disminuyendo significativamente la densidad de los cenobios más grandes. Además, los info-químicos indujeron un menor incremento de los cenobios más grandes comparados con el control (la tasa de cambio fue un $45 \%$ menor que en los cultivos control). Nuestro estudio sugiere que el tamaño del cenobio de P. tetras está sometido a un posible compromiso (trade-off) en su medio natural: la población de P. tetras tendería a incrementar su tamaño para una mejor asimilación de los nutrientes y a disminuirlo cuando grandes herbívoros están presentes.

Palabras clave: Formación de cenobios, Daphnia magna, info-químicos.

\section{INTRODUCCIÓN}

La relación entre tamaño y forma corporal (forma geométrica, presencia de espinas, organización del cenobio, etc.) de las microalgas en sus diferentes períodos de crecimiento es bien conocida desde hace tiempo. Por ejemplo, variaciones en los tricomas de Limnothrix redeckei (Van Goor) Meffert (Meffert \& Oberhauser, 1982) o formación de colonias o cenobios de Pediastrum (Davis, 1967; 
Millington \& Rasch, 1980). Además, se conocen cambios en la morfología debidos a factores abióticos tales como la temperatura (Lürling \& Van Donk, 1999), los nutrientes (Verity \& Medlin, 2003) y la estabilidad relativa de la columna de agua (Naselli-Flores \& Barone, 2003), o debidos a factores bióticos como la depredación (Wiltshire et al., 2003), los info-químicos de depredadores (Tang, 2003), la competencia (Yoshida et al., 2004) o la alelopatía (Mulderij et al., 2005) y debidos al efecto combinado de factores bióticos y abióticos (Lürling \& Van Donk, 1999).

La variabilidad y plasticidad morfológica en poblaciones de fitoplancton no sólo son características relevantes para la taxonomía, sistemática o estudios de evolución (Chang \& Chang-Schneider, 1980; Michod et al., 2003; Shubert \& Wilk-Wozniak, 2003), sino que además son características clave en procesos ecológicos del plancton como, por ejemplo, la producción acuática (Kerr \& Dickie, 2001). Los cambios morfológicos de una población pueden ser debidos a su propio ciclo de vida o a la influencia de los factores ambientales, pero además, estos cambios pueden deberse a variaciones en la comunidad planctónica. La bibliografía más reciente sobre plasticidad morfológica explica la manera en la que esto se produce, por ejemplo, la morfología es determinante en la relación de depredación (Mayeli et al., 2004), en la función de fijación de nitrógeno (Chan et $a l .$, 2004), en la producción de toxinas (Kurmayer et al., 2003), en las necesidades metabólicas (Li \& Gao, 2004) o la capacidad de flotación (Padisák et al., 2003; Peperzak et al., 2003).

No obstante, a pesar de la relevancia de la forma del fitoplancton para la ecología (Reynolds, 1997), muchas algas no han sido aisladas y cultivadas, por ello su respuesta morfológica frente a condiciones ambientales sigue siendo desconocida. De hecho, los estudios arriba mencionados están restringidos a unas pocas especies de los géneros Phaeocystis (Lagerheim), Microcystis (Kützing ex Lemmermann), Anabaena (St. Vincent ex Bornet \& Flash), Scenedesmus (Meyen), Staurastrum (Meyen),
Pediastrum, Nostoc (Vaucherex Bornet \& Flahault), y los más estudiados son aquellos capaces de formar "blooms" como Phaeocystis, Microcystis, Anabaena o Scenedesmus (véase, por ejemplo, Veldhuis et al., 1986; Roelke \& Buyucates, 2002; Faithfull \& Burns, 2006). Scenedesmus, alga verde cocal no móvil, produce una sucesión de formas en cultivo y su plasticidad fenotípica ha sido demostrada cuando las condiciones ambientales del cultivo se alteran (Chang \& Chang-Schneider, 1980; Trainor, 1993). Además, las estrategias de defensa de las algas frente a depredadores también producen cambios morfológicos. Este mecanismo ha sido estudiado, generalmente, en el sistema Scenedesmus-Daphnia (Hessen \& Van Donk, 1993; Lürling \& Van Donk, 1999) y no parece claro en otros grupos de algas (Wiltshire et al., 2003). Esta es la razón por la que los estudios sobre la formación de cenobios en poblaciones aisladas son útiles para destacar las posibilidades en expresión morfológica de las algas y obtener respuestas sobre la ecología de algas similares.

Cuando la población estudiada presenta plasticidad fenotípica surge una pregunta: ¿Cómo puede inducir el ambiente la aparición de uno u otro tamaño/forma en la distribución de la población?. En este estudio, se ha usado un cultivo de Pediastrum tetras (Ehrenberg) Ralfs para conocer los cambios en la estructura de la población provocados por la depredación por Daphnia magna (Straus) y por la variación en la concentración de nutrientes. Se decidió utilizar una especie de Pediastrum porque es un género cosmopolita y su biomasa es relevante en numerosos cuerpos de agua (Tiwari \& Chauhan, 2006; Komárek \& Jankovska, 2001), aunque sólo con $P$. boryanum y $P$. simplex se han realizado estudios de plasticidad más detallados (Davis, 1967; Chang \& ChangSchneider, 1980). Además, $P$. tetras presenta cenobios con diferentes números de células, siendo la especie de este género que forma los cenobios más pequeños (cenobios de cuatro células) (Fritsch, 1935; Komárek \& Jankovská, 2001). 


\section{MATERIAL Y MÉTODOS}

\section{Cultivo algal}

La cepa de $P$. tetras utilizada para estudiar las diferencias morfológicas producidas por factores ambientales procedía de la Culture Collection of Algae and Protozoa (CCAP, número de referencia 261/8, Reino Unido). Esta población se mantuvo como stock en el laboratorio en un cultivo semi-continuo usando dos matraces de $50 \mathrm{ml}$ con $25 \mathrm{ml}$ de medio Jaworski (recomendado por CCAP). $1 \mathrm{ml}$ del cultivo stock de $P$. tetras se transfería a $25 \mathrm{ml}$ de nuevo medio de cultivo cada dos semanas. El cultivo se encontraba en una cámara climática controlada a $20^{\circ} \mathrm{C}$, iluminada con una irradiancia PAR de $30 \mu \mathrm{E} \mathrm{m}^{-2} \mathrm{~s}^{-1}$ con un ciclo 12:12h luz:oscuridad. El cultivo se agitaba manualmente dos veces por semana y la población era regularmente examinada mediante un microscopio invertido. El inóculo utilizado en los experimentos fue tomado de este cultivo stock de $P$. tetras.

\section{Cultivo de Daphnia magna}

D. magna se aisló en 2003 del lago de la Albufera (Valencia, España), y se mantuvo como un stock en el laboratorio mediante un cultivo semi-continuo usando matraces de $250 \mathrm{ml}$, los cuales contenían $150 \mathrm{ml}$ de agua mineral y $5 \mathrm{ml}$ de un cultivo de Monoraphidium contortum (Thur.) Kom.-Legn. (aproximadamente en una concentración de $10^{5}$ células $/ \mathrm{ml}$ ). Cada semana 8 individuos de D. magna se transferían a un nuevo medio de cultivo. Las condiciones de cultivo eran las mismas que las descritas para el cultivo algal. Utilizamos este stock para obtener los ejemplares de D. magna que necesitábamos para los experimentos.

\section{Experimentos sobre factores de control de la variabilidad morfológica de la población}

Estos experimentos contaban con tres tratamientos y un control, los cuales estaban constituidos por $2 \mathrm{ml}$ de cultivo stock de P. tetras, el cual se encontraba en el día 15 desde el momento en el que fue inoculado, $20 \mathrm{ml}$ de agua mineral (control), agua mineral con D. magna (tratamiento de depredación), agua mineral con un suministro de nutrientes (tratamiento de nutrientes) y agua con info-químicos generados por D. magna (tratamiento con info-químicos); todas estas experiencias se realizaron en matraces de $50 \mathrm{ml}$ y por triplicado. Se sabe por la literatura científica que otros experimentos sobre los efectos de la depredación de distintas especies de Daphnia o la presencia de info-químicos sobre algas usan períodos de tiempo de experimentación que varían en un amplio rango. Así, por ejemplo, Wiltshire et al. (2003) utilizan 4 horas frente a Lürling et al. (1997) y Lürling \& Van Donk (1999) que emplean 2 días. Nosotros decidimos finalizar los experimentos tras 5 días de cultivo añadiendo unas gotas de Lugol en los matraces.

Para el estudio de los posibles efectos que estos factores podrían inducir en la estructura de la población de $P$. tetras se observaron y recontaron muestras tomadas de los distintos tratamientos de la siguiente manera: se tomó $1 \mathrm{ml}$ de cultivo cada 2 días de cada uno de los tratamientos y se observaron, previa sedimentación en cámaras Utermöhl, en un microscopio invertido Olympus a 400 X. Para cada muestra, observamos y contamos: i) el número de los diferentes cenobios (desde una célula aislada hasta cenobios de 16 células; cenobios de más de 16 células fueron poco comunes) y ii) el número de cenobios grandes y pequeños para cada tipo de cenobio. Se contaron más de 400 células en cada muestra con el fin de reducir el error de recuento (Lund et al., 1958). Tanto de las células aisladas como de cada tipo de cenobio se midió la dimensión mayor (GALD) de más de cien ejemplares.

El experimento sobre la depredación se llevó a cabo para conocer el efecto de D. magna sobre P. tetras, buscando no sólo un aclarado en el agua del matraz debido a la depredación sino más bien un cambio en la densidad relativa de los diferentes tipos de cenobios. Este tratamiento se realizó con $20 \mathrm{ml}$ de agua mineral y cuatro individuos de D. magna de tamaño corporal medio $(1.69 \pm 0.03 \mathrm{~mm}$ ) (para prevenir nuevos nacimientos de D. magna). Los individuos de D. magna fueron aclimatados 
previamente permitiendo que pudiesen vivir tres días en un cultivo con P. tetras como único alimento $(0.5 \mathrm{mg}$ peso seco/l).

Para conocer los posibles cambios en la formación de cenobios inducidos por químicos excretados por $D$. magna o si ésta necesitaba estar presente en el cultivo para inducir los cambios, el alga fue cultivada en agua donde había habido previamente una densa población de $D$. magna (330 ind/l) durante 7 días, periodo en el que había sido alimentada con $P$. tetras. Se trata de una concentración de $D$. magna equivalente a $5.4 \mathrm{mg}$ peso seco/l, concentración mayor que los $0.5 \mathrm{mg}$ peso seco/l que puede afectar a Scenedesmus (Lampert et al., 1994). Este cultivo fue filtrado a través de una membrana de $0.2 \mu \mathrm{m}$ de diámetro de poro; el filtrado obtenido, el cual supuestamente contenía los info-químicos producidos por D. magna, fue utilizado para el cultivo de $P$. tetras. La concentración de nutrientes de este agua fue de $0.014 \mathrm{mg} \mathrm{P}_{-} \mathrm{PO}_{4} / \mathrm{l}$ y el $\mathrm{NO}_{3}$ fue indetectable, similar a la baja concentración de nutrientes que posee el agua mineral.

Finalmente, el efecto de la disponibilidad de nutrientes en el crecimiento y distribución de los cenobios de $P$. tetras fue analizado incrementando la concentración de nutrientes: $2 \mathrm{ml}$ del cultivo algal fueron inoculados en $16 \mathrm{ml}$ de agua mineral y se añadieron $4 \mathrm{ml}$ de medio de cultivo (concentración final de nutrientes: $1.18 \mathrm{mg}$ P-PO $-1 / \mathrm{l}$ y $\left.2.88 \mathrm{mg} \mathrm{N}-\mathrm{NO}_{3} / \mathrm{l}\right)$.

La tasa de cambio de la densidad celular de $P$. tetras para cada tipo de cenobio fue calculada con la siguiente ecuación:

$$
\text { Tasa cambio }=\left(D_{5}-D_{0}\right) /\left(D_{0} * t\right)
$$

donde, $D_{5}$ es el número de células en el día $5, D_{0}$ número de células en el día 0 y $t$ es tiempo de experimentación (5 días).

\section{Métodos estadísticos}

Se utilizaron análisis de la varianza (ANOVA) de una vía para comparar la tasa de cambio de la densidad final de $P$. tetras en los diferentes tratamientos y de los diferentes tipos de formas que presentaba el alga bajo los
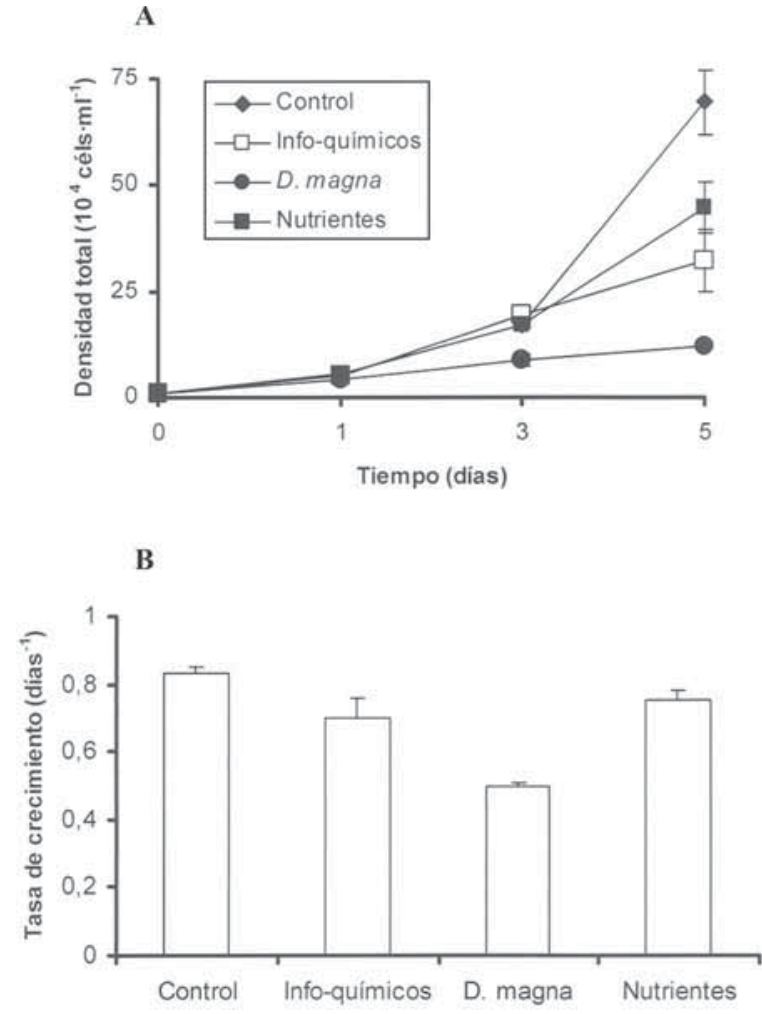

Figura 1. A. Densidad total de células de P. tetras en los tres tratamientos y en el control. B. Tasa de crecimiento (días ${ }^{-1}$ ) en los cinco primeros días del cultivo de P. tetras en los tres tratamientos y en el control. Las barras representan el error estándar. A. Total cell density of P. tetras in the three treatments and the control. B. Growth rate (days ${ }^{-1}$ ) in the first five days of P. tetras' culture in the three treatments and the control. The bars represent the standard error.

mismos tratamientos. Se consultó el test Levene de homogeneidad de varianzas, seguido del test de comparación post hoc de Bonferroni para conocer qué tratamientos eran significativamente diferentes con $p=0.05$. Todos los análisis estadísticos se realizaron mediante la herramienta estadística SPSS versión 13.0.

\section{RESULTADOS}

Los resultados de los diferentes tratamientos estudiados (depredación por D. magna, el efecto de info-químicos y el incremento en la concentración de nutrientes) indican que $P$. tetras crece mejor en primer lugar, en un cultivo con agua mineral únicamente (control), seguido del cul- 
Tabla 1. Promedio de la dimensión lineal axial mayor (GALD, $\mu \mathrm{m} \pm$ error estándar) de células y cenobios de $P$. tetras entre tratamientos, se indica el valor mínimo y máximo observado durante la experiencia. Se midió la dimensión mayor de al menos 100 cenobios de cada tipo. Average greatest axial linear dimension (GALD, $\mu m \pm$ standard error) of P. tetras cells and coenobiums between treatments. Minimum and maximum values that were observed during the experience are shown. The longest dimension of at least 100 colonies of each type was measured.

\begin{tabular}{|c|c|c|c|c|c|c|c|c|}
\hline & \multicolumn{2}{|c|}{ Control } & \multicolumn{2}{c|}{ Info-químicos } & \multicolumn{2}{c|}{ D. magna } & \multicolumn{2}{c|}{ Nutrientes } \\
\hline & min. & máx. & min. & máx. & min. & máx. & min. & máx. \\
\hline Célula aislada & $10 \pm 0.6$ & $13 \pm 0.0$ & $10 \pm 1.0$ & $13 \pm 0.0$ & $10 \pm 0.6$ & $13 \pm 0.0$ & $8 \pm 0.0$ & $13 \pm 0.0$ \\
\hline Cenobio de 4 cél. & $17 \pm 0.6$ & $23 \pm 0.0$ & $17 \pm 4.0$ & $23 \pm 0.0$ & $18 \pm 4.0$ & $23 \pm 0.0$ & $16 \pm 0.6$ & $25 \pm 0.0$ \\
\hline Cenobio de 8 cél. & $20 \pm 0.6$ & $29 \pm 0.0$ & $21 \pm 1.0$ & $29 \pm 0.0$ & $23 \pm 1.2$ & $29 \pm 0.0$ & $23 \pm 1.2$ & $29 \pm 0.0$ \\
\hline Cenobio de 16 cél. & $32 \pm 1.2$ & $41 \pm 0.0$ & $31 \pm 1.0$ & $41 \pm 0.0$ & $32 \pm 1.2$ & $41 \pm 0.0$ & $33 \pm 2.3$ & $41 \pm 0.0$ \\
\hline
\end{tabular}

tivo con agua más un suministro de nutrientes, después en agua donde previamente había estado D. magna y, finalmente, en un cultivo con individuos de D. magna como depredadores (Fig. 1.A). Los tamaños alcanzados por las diferentes formas (célula simple o cenobios) en los distintos tratamientos se indican en la Tabla 1. La ANOVA de una vía y el test post hoc de Bonferroni mostraron que sólo la tasa de crecimiento de P. tetras en presencia de D. magna fue significativamente diferente en relación a los otros dos tratamientos ( $p=0.022$ para de info-químicos, $p=0.006$ para nutrientes) y el control ( $p=0.001)$. Los otros dos

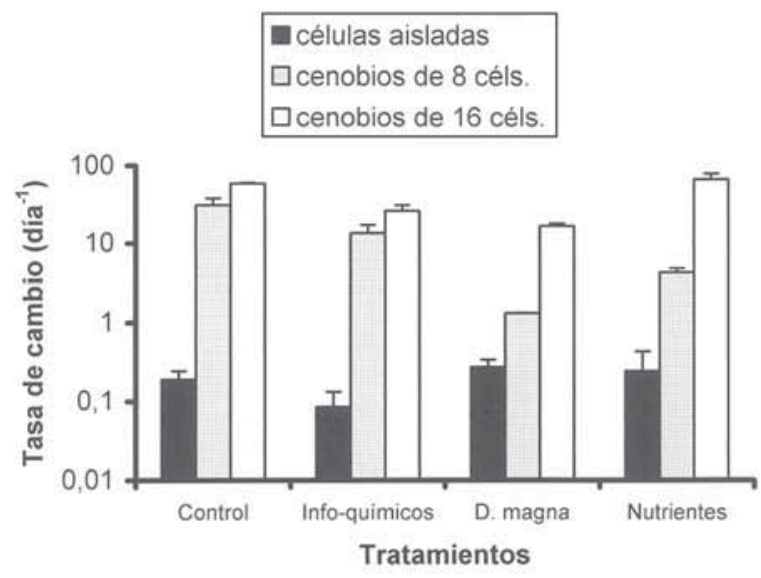

Figura 2. Media y error estándar de la tasa de cambio de la densidad (días ${ }^{-1}$, incremento per capita en 5 días en cel $/ \mathrm{ml}$ ) de células aisladas y de los dos tipos de cenobios más representativos (cenobios de 8 y 16 células) en los diferentes tratamientos y en el control. Average and standard error of the density' rate of change (days ${ }^{-1}$, per capita increase in 5 days in cells $/ \mathrm{ml}$ ) of isolated cells and the two most representative coenobium types (8- and 16-celled coenobium) in the different treatments and control. tratamientos y el control no resultaron ser signi ficativamente diferentes, de esta manera, la posible presencia de info-químicos y el suministro de nutrientes no varía significativamente la tasa de crecimiento de $P$. tetras. (Fig. 1.B)

El incremento de densidad de los diferentes tipos de cenobios no se ajusta a una función exponencial, por lo que calculamos la tasa de cambio en células/ml de los diferentes tipos de cenobios y se compararon las medias mediante ANOVA de una vía (Tabla 2). Se observó que las células aisladas o los distintos tipos de cenobios aumentaban ligeramente, pero con una proporción muy similar en todos los tratamientos y el control (Fig. 2 y Tabla 2). Los cenobios de 4 células mostraron el mismo comportamiento. En cenobios formados por 8 células se pudo ver que la tasa de cambio fue significativamente inferior en presencia de D. magna y con un suministro de nutrientes (Tabla 2), mostrando, aparentemente, dos grupos de efectos: ControlInfo-químicos diferente a D. magna-Nutrientes. Sin embargo, cuando comparamos la tasa de cambio de los cenobios de 16 células en los diferentes tratamientos, Control y Nutrientes no mostraron diferencias significativas, así el suministro de nutrientes no parece afectar a esta forma de cenobio. Además, la presencia de D. magna y los posibles info-químicos presentes en el cultivo provocaron una tasa de cambio inferior a la producida en el control o en cultivos con suministro de nutrientes (Fig. 2 y Tabla 2). De esta manera, observamos dos posibles grupos de efectos: Control-Nutrientes versus D. magna-Info-químicos. 
Tabla 2. ANOVA de una vía de la tasa de cambio de la densidad $\left(\operatorname{dias}^{-1}\right)$ comparando los tratamientos con el control. Se indican las diferencias significativas como probabilidades y "ns" se refiere a diferencias no significativas. One-way ANOVA on the density's rate of change $\left(\right.$ days $\left.^{-1}\right)$ comparing the treatments with the control. Significant differences are indicated as probabilities and " $n s$ " means non-significant differences.

\begin{tabular}{|c|c|c|c|c|}
\hline ANOVA de una vía & Tratamientos & versus & Control & \\
\hline & Célula aislada & Cenobio de 4 cél. & Cenobio de 8 cél. & Cenobio de 16 cél. \\
\hline Info-químicos & ns & ns & ns & 0.002 \\
\hline D. magna & ns & $\mathrm{ns}$ & 0.012 & 0.000 \\
\hline Nutrientes & $\mathrm{ns}$ & $\mathrm{ns}$ & 0.017 & $\mathrm{~ns}$ \\
\hline \multicolumn{5}{|c|}{ Test post hoc de Bonferroni } \\
\hline Control/Info-químicos & $\mathrm{ns}$ & $\mathrm{ns}$ & ns & 0.038 \\
\hline Control/D. magna & $\mathrm{ns}$ & $\mathrm{ns}$ & 0.004 & 0.009 \\
\hline Control/Nutrientes & ns & ns & 0.007 & ns \\
\hline Info-químicos/Nutrientes & ns & ns & ns & 0.012 \\
\hline D. magna/Nutrientes & ns & ns & ns & 0.003 \\
\hline
\end{tabular}

\section{DISCUSIÓN}

El principal objetivo de este estudio fue entender cómo la estructura de una población de cenobios puede verse afectada por factores externos. Parece ser que factores externos al propio cultivo de P.tetras pueden controlar la estructura del cenobio, como por ejemplo, la concentración de nutrientes o la presión por depredación selectiva. A pesar del hecho de que P.tetras es una especie común en lagos mesotróficos y eutróficos (Komárek \& Jakovská, 2001), sus cenobios de gran tamaño se asocian también a un bajo estado trófico (Nasselli-Flores \& Barone 2000). En este estudio, el incremento en la concentración de nutrientes no varió la tasa de crecimiento de la población y la densidad de algunos tipos de cenobios (ej. los cenobios de tamaño medio) aumentaron menos que en el control. Esto es sólo una demostración del efecto de los nutrientes sobre la estructura de una población, pero apenas explica la complejidad en la absorción idónea de nutrientes como, por ejemplo, se conoce en el género Phaeocystis (Ploug et al., 1999; Whipple et al., 2005). Los integrantes del mesozooplancton, como D. magna, no solo contribuyen significativamente en la presión de depredación sobre el fitoplancton (Sommer et al.,
1986) sino que además cambian la estructura del tamaño del fitoplancton (Sommer et al., 2001). Esto es debido a que los Cladóceros se alimentan selectivamente de un rango de tamaños (Gliwicz, 1969, 1980), de formas (Nadin-Hurley \& Duncan, 1976) y de calidad del alimento (Lürling et al., 1997). A pesar de que D. magna es un eficiente depredador de alimento de pequeño tamaño, como una bacteria (Gophen \& Séller, 1984; Gulati et al., 2001), los individuos de tamaño corporal medio ingieren partículas de hasta, aproximadamente, $50 \mu \mathrm{m}$ GALD (Burns, 1968). Por tanto, es probable que D. magna pueda consumir los cenobios más grandes de P. tetras, como ha sido demostrado en este estudio. Además, existen rangos óptimos de tamaño de alimento para Cladóceros, por ejemplo, D. magna, con una longitud de 0.5 a $1 \mathrm{~mm}$ muestra una selección de tamaño óptimo de 11 a $23 \mu \mathrm{m}$ (Gliwicz, 1969, 1980).

Otros resultados muestran que D. magna es capaz de seleccionar grandes partículas (Jensen et al., 2001). En nuestro estudio, los individuos de tamaño medio de D. magna afectaban selectivamente a los cenobios grandes de P. tetras (de 8 y 16 células, de 20 a $41 \mu \mathrm{m}$ ) y no fue evidente un efecto de depredación sobre cenobios de pocas células. Hay que destacar la importancia de 
la filtración selectiva que se ejerce sobre microalgas, la cual, en este caso, parece favorecer a los cenobios pequeños y afectar a los grandes.

Otro efecto que se puede observar en la estructura de P.tetras es el efecto indirecto del depredador debido a la producción de infoquímicos los cuales reducen la frecuencia de aparición de nuevos cenobios grandes. Desde el trabajo pionero de Hessen \& van Donk (1993), la importancia de la respuesta adaptativa de las microalgas frente a los info-químicos de los depredadores es un tema prolífico. Se ha sugerido que la formación de los cenobios, cuando la especie es polimórfica, puede ser una defensa frente a la depredación (Dodson, 1989). Este es el caso de, por ejemplo, Scenedesmus y Phaeocystis (Verity \& Medlin, 2003; Tang, 2003; Verschoor et al., 2004), se conoce bien que la existencia de info-químicos de depredadores incrementa la presencia de las formas más grandes en sus poblaciones. Éstas llegan a tener más relevancia en la población, incluso cuando todavía siguen siendo comestibles por los depredadores. De ahí que el factor que induce la formación del cenobio provoque una respuesta fisiológica general independiente del tamaño actual (Lampert et al., 1994). Sin embargo, en nuestro estudio, los info-químicos promovieron la reducción del número de cenobios grandes. Esto puede tener un significado adaptativo similar ya que parece probado que D. magna se alimenta selectivamente de los cenobios de P. tetras de mayor tamaño. En Scenedesmus, los químicos inducen mecanismos por los cuales las células en división permanecen adheridas al abandonar la célula madre (Trainor, 1993). Esto puede sugerir que los info-químicos impiden la formación de nuevos cenobios de 16 células, los cuales pueden proceder de la fase sexual o asexual del ciclo de vida del alga (observación personal). Por tanto, la cuestión sobre si otras algas clorococales, como Scenedesmus, muestran respuestas frente a factores químicos (Lampert et al., 1994) está respondida: el cenobio de $P$. tetras es sensible a info-químicos, pero de forma diferente a lo que lo es Scenedesmaceae, donde la presencia de info-químicos induce una mayor presencia de cenobios de gran tamaño.
A modo de conclusiones podemos resumir los aspectos más relevantes aquí explicados:

- La imagen que teníamos sobre la plasticidad fenotípica de $P$. tetras es que las formas pequeñas son más abundantes cuando existe un incremento en la concentración de nutrientes (nuevo medio fresco o un lago eutrófico). Este es un comportamiento algal bien conocido a favor de la estrategia de la " $r$ " (Reynolds, 1997; Sommer et al., 1986). Cuando los nutrientes en el medio de cultivo se agotan o el lago tiene pocos nutrientes, entonces los cenobios grandes son más frecuentes (Naselli-Flores \& Barone, 2000), y el crecimiento de la población se basa en la formación de nuevos cenobios grandes, es decir, sigue una estrategia de la " $K$ ".

- El compromiso ("trade-off”) entre la pérdida por sedimentación y la resistencia a la depredación, discutido en el caso de Scenedesmaceae o Phaeocystis (Lampert et al., 1994; Whipple et al., 2005), parece irrelevante para P.tetras.

- La formación de cenobios grandes de P. tetras no se produce, de forma fenotípica, cuando las señales químicas en el medio indican la presencia de grandes depredadores, sin el coste de la reducción de la tasa de crecimiento $\mathrm{y}$, además, reduciendo las posibles pérdidas por sedimentación de las formas grandes (Padisák et al., 2003) en la estratificación del período cálido cuando la depredación es más relevante (Sommer et al., 1986). Por tanto, sugerimos otro posible compromiso entre opciones: durante el periodo estable en un lago, la población de $P$. tetras tenderá a incrementar su tamaño para asimilar mejor los nutrientes y lo disminuirá cuando existan grandes herbívoros presentes en el medio.

- A partir del estudio aquí realizado se ha profundizado en el conocimiento de la estructura de tamaños de la población de P.tetras, pero quedan todavía muchas cuestiones por abordar, como por ejemplo, comprobar si otras especies de Pediastrum, con pocas formas pequeñas, como P. boryanum, presentan respuestas parecidas. 


\section{AGRADECIMIENTOS}

Los autores quieren agradecer al Ministerio de Educación y Ciencia por la financiación del proyecto CGL2006-02891. Este experimento se realizó gracias a la beca concedida a Matilde Segura por la Universidad de Valencia. Nos gustaría agradecer a Jose Larrosa su ayuda al inicio de la experiencia y a Augusto Comas (en Cuba), Miguel Álvarez-Cobelas (en España) y los cuidados revisores del artículo por las sugerencias que nos aportaron.

\section{REFERENCES}

BURNS, C. W. 1968. The relationship between body size of filter-feeding Cladocera and the maximum size of particle ingested. Limnol. Oceanogr., 13: 675-678.

CHAN, F., M. L. PACE, R. W. HOWARTH \& R. M. MARINO. 2004. Bloom formation in heterocystic nitrogen-fixing cyanobacteria: The dependence on colony size and zooplankton grazing. Limnol. Oceanog., 49: 2171-2178.

CHANG, V. T. \& H. CHANG-SCHNEIDER. 1980. Zur Variabilität von Pediastrum boryanum (Turpin) Meneghini. Algological Studies, 26: 53-62.

CHANG, T. P. 1981. A comparative study of Pediastrum boryanum (Turp) Menegh and Pediastrum duplex Meyen. Archiv fur Protistenkunde, 124: 232-243.

DAVIS, J. S. 1967. The life cycle of Pediastrum simplex. J. Phycol., 3: 95-103.

DODSON, S. 1989. Predator-induced reaction norms. BioScience, 39:447-452.

FAITHFULL, C. L. \& C. W. BURNS. 2006. Effects of salinity and source of inocula on germination of Anabaena akinetes from a tidally influenced lake. Freshwat. Biol., 51: 705-716

FRITSCH, F. E. 1935. The structure and reproduction of the algae. Cambridge University Press, Cambridge. $809 \mathrm{pp}$.

GLIWICZ, Z. M. 1969. Studies on the feeding of pelagic zooplankton in lakes with varying trophy. Ekol. Pol., 17: 665-676.

GLIWICZ, Z. M. 1980. Filtering rates, food size selection, and feeding rates cladocerans- another aspect of interspecific competition in filter-feeding zooplankton. In: Evolution and Ecology of Zooplankton Communities. W. C. Kerfoot (ed.): 282291. University Press of New England, Hanover.

GOPHEN, M. \& W. GELLER. 1984. Filter mesh size and food particle uptake by Daphnia. Oecologia, 64: 408-412.

GULATI, R. D., M. BRONKHORST \& E. VAN DONK. 2001. Feeding in Daphnia galeata on Oscillatoria limnetica and on detritus derived from it. J. Plankton Res., 23: 705-718.

HESSEN, D. \& E. VAN DONK. 1993. Morphological changes in Scenedesmus induced by substances released from Daphnia. Arch. Hydrobiol., 127: 129-140.

JENSEN, T. C., D. O. HESSEN \& B. A. FAAFENG. 2001. Biotic and abiotic preferences of the cladocerans invader Limnosiga frontosa. Hydrobiologia, 442: 89-99.

KERR, S. R. \& L. M. DICKIE. 2001. The biomass spectrum. A predator-prey theory of aquatic production. Columbia University Press, New York. 352 pp.

KOMÁREK, J. \& V. JANKOVSKÁ. 2001. Review of the Green Algal Genus Pediastrum: Implications for Pollen-analytical Research. Bibliotheca Phycologica, 108. 127 pp. Cramer J., Berlin-Stuttgart.

KURMAYER, R., G. CHRISTIANSEN \& I. CHORUS. 2003. The abundance of microcystinproducing genotypes correlates positively with colony size in Microcystis sp. and determines its microcystin net production in Lake Wannsee. Appl. Environ. Microbiol., 69: 787-795.

LAMPERT, W., K. O. ROTHHAUPT \& E. VON ELERT. 1994. Chemical induction of colony formation in a green alga (Scenedesmus acutus) by grazers (Daphnia). Limnol. Oceanogr., 39: 15431550.

LI, Y. G. \& K. S. GAO. 2004. Photosynthetic physiology and growth as a function of colony size in the cyanobacterium Nostoc spaheroides. Eur. J. Phycol., 39: 9-15.

LUND, J. W. G., C. KIPLING \& E. D. LE CREN. 1958. The inverted microscope method of estimating algal numbers and statistical basis of estimations by counting. Hydrobiologia, 11: 143-170.

LÜRLING, M., H. J. DE LANGE \& E. VAN DONK. 1997. Changes in food quality of the green alga Scenedesmus induced by Daphnia info-chemicals: biochemical composition and morphology. Freshwat. Biol., 38: 619-628. 
LÜRLING, M. \& E. VAN DONK. 1999. Grazer-induced colony formation in Scenedesmus acutus (Chlorophyceae): ecomorph expression at different temperatures. J. Phycol., 35: 1120-1126.

MAYELI, S. M., S. NANDINI \& S. S. S. SARMA. 2004. The efficacy of Scenedesmus morphology as a defense mechanism against grazing by selected species of rotifers and cladocerans. Aquatic Ecology, 38: 515-524.

MEFFERT, M. E. \& R. OBERHAUSER. 1982. Polar and centric gas vacuoles in planktonic Oscillatoria species (Cyanophyta). Arch. Hydrobiol., 95: 235248.

MICHOD, R. E., A. N. NEDELCU \& D. ROZEE. 2003. Co-operation and conflict in the evolution of individuality IV. Conflict mediation and evolvability in Volvox carteri. BioSystem, 69: 95-114.

MILLINGTON, W. F. \& E. M. RASCH. 1980. Microspectrophotometric analysis of mitosis and DNA synthesis associated with colony formation in Pediastrum boryanum Chlorophyceae). J. Phycol., 16:177-182

MULDERIJ, G., W. M. MOOIJ \& VAN DONK, E. 2005. Allelopathic growth inhibition and colony formation of the green alga Scenedesmus obliquus by the aquatic macrophyte Stratiotes aloides. Aquatic Ecology, 39: 11-21.

NADIN-HURLEY, C. \& A. DUNCAN. 1976. A comparison of daphnid gut particles with the sestonic present in two Thames Valley reservoirs throughout 1970 and 1971. Freshwat. Biol., 6: 109123.

NASELLI-FLORES, L. \& R. BARONE. 2000. Phytoplankton dynamics and structure: a comparative analysis in natural and man-made water bodies of different trophic state. Hydrobiologia, 438: 65-74.

NASSELLI-FLORES, L. \& R. BARONE. 2003. Steady-state assemblages in a Mediterranean hypertrophic reservoir. The role of Microcystis ecomorphological variability in maintaining an apparent equilibrium. Hydrobiologia, 502: 133-143.

PADISÁK, J., É. SORÓCZKI-PINTÉR \& Z. REZNER. 2003. Sinking properties of some phytoplankton shapes and the relation of form resistance to morphological diversity of plankton -an experimental study. Hydrobiologia, 500: 243-257.

PEPERZAK, L., E. COLIJN, R. KOEMAN, W. W. C. GIESKES \& J. C. A. JOORDENS. 2003. Phytoplankton sinking rates in the Rhine region of freshwater influence. J. Plankton Res., 25: 365-383.
PLOUG, H., W. STOLTE \& B. B. JØRGENSEN. 1999. Diffusive boundary layers of the colonyforming plankton alga Phaeocystis sp.- implications for nutrient uptake and cellular growth. Limnol. Oceanogr., 44: 1957-1967.

REYNOLDS, C. S. 1997. Vegetation Processes in the Pelagic: A model for ecosystem theory. Ecology Institute, Oldendorf. $371 \mathrm{pp}$.

ROELKE, D. \& Y. BUYUCATES. 2002. Dynamics of phytoplankton succession coupled to species diversity as a system-level tool for study of Microcystis population dynamics in eutrophic lakes. Limnol. Oceanogr., 47: 1109-1118.

SHUBERT, L. E. \& E. WILK-WOZNIAK. 2003. SEM investigation of several non-motile coccoid green algae isolated from aquatic habitats in Poland. Biologia Bratislava, 58: 459-466.

SOMMER, U., Z. M. GLIWICZ, W. LAMPERT \& A. DUNCAN. 1986. The PEG-model of seasonal succession of planktonic events in lakes. Arch. Hydrobiol., 106: 433-471.

SOMMER, U., F. SOMMER, B. SANTER, C. JAMIESON, M. BOERSMA, C. BECKER \& T. HANSEN. 2001. Complementary impact of copepods and cladocerans on phytoplankton. Ecol. Lett., 4: 545-550.

TANG, K. W. 2003. Grazing and colony size development in Phaeocystis globosa (Prymnesiophyceae): the role of chemical signal. J. Plankton. Res., 25: 831-842.

TIWARI, A. \& S. V. S. CHAUHAN. 2006. Seasonal phytoplanktonic diversity of Kitharn lake, Agra. J. Environ. Biol., 27: 35-38.

TRAINOR, F. R. 1993. Cyclomorphosis in Scenedesmus subspicaus (Chlorococcales, Chlorophyta): stimulation of colony development at low temperature. Phycologia, 32: 429-433.

VELDHUIS, M. J. W., F. COLIJN \& L. A. H. VENEKAMP. 1986. The spring bloom of Phaeocystis pouchetii (Haptophyceae) in Dutch coastal waters. Neth. J. Sea Res., 20: 37-48.

VERITY, P. G. \& L. K. MEDLIN. 2003. Observations on colony formation by the cosmopolitan phytoplankton genus Phaeocystis. J. Marine Syst., 43: 153- 164.

VERSCHOOR, A. M., I. VAN DER STAP, N. R. HELMSING, M. LÜRLING \& E. VAN DONK. 2004 Inducible colony formation within the Scenedesmaceae: Adaptive responses to infochemicals from two different herbivore taxa. $J$. Phycol., 40: 808-814. 
WHIPPLE, S. J., B. C. PATTERN \& P. G. VERITY. 2005. Life cycle of marine alga Phaeocystis: A conceptual model to summarize literature and guide research. Journal of marine systems, 57: 83-110.

WILTSHIRE, K., M. BOERSMA \& B. MEYER. 2003. Grazer-induced changes in desmid Stauras- trum. Hydrobiologia, 491: 255-260.

YOSHIDA, T., N. G. HAIRSTON, J.R. \& S. P. ELLNER. 2004. Evolutionary trade-off between defence against grazing and competitive ability in a simple unicellular alga, Chlorella vulgaris. Proc. R. Soc. Lond., 271: 1947-1953. 